\title{
CRITICAL CURVES FOR A COUPLED SYSTEM OF FAST DIFFUSIVE NEWTONIAN FILTRATION EQUATIONS
}

\author{
RUNMEI DU and ZEJIA WANG ${ }^{凶}$
}

(Received 13 November 2011)

\begin{abstract}
This paper deals with the large-time behaviour of solutions to the fast diffusive Newtonian filtration equations coupled via the nonlinear boundary sources. A result of Fujita type is obtained by constructing various kinds of upper and lower solutions. In particular, it is shown that the critical global existence curve and the critical Fujita curve concide for the multi-dimensional system. This is quite different from the known results obtained in Wang, Zhou and Lou ['Critical exponents for porous medium systems coupled via nonlinear boundary flux', Nonlinear Anal. 7(1) (2009), 2134-2140] for the corresponding one-dimensional problem.
\end{abstract}

2010 Mathematics subject classification: primary 35K65; secondary 35B33.

Keywords and phrases: global existence, blow-up, Newtonian filtration equation, critical curve.

\section{Introduction}

In this paper, we study the system

$$
\begin{gathered}
\frac{\partial u}{\partial t}=\Delta u^{m}, \quad \frac{\partial v}{\partial t}=\Delta v^{n}, \quad(x, t) \in\left(\mathbb{R}^{N} \backslash B_{1}(0)\right) \times(0, T), \\
\nabla u^{m} \cdot \vec{v}=v^{p}(x, t), \quad \nabla v^{n} \cdot \vec{v}=u^{q}(x, t), \quad(x, t) \in \partial B_{1}(0) \times(0, T), \\
u(x, 0)=u_{0}(x), \quad v(x, 0)=v_{0}(x), \quad x \in \mathbb{R}^{N} \backslash B_{1}(0),
\end{gathered}
$$

where $0<m, n<1, p, q \geq 0, N>2, B_{1}(0)$ is the unit ball in $\mathbb{R}^{N}, \vec{v}$ is the inward normal vector on $\partial B_{1}(0)$, and $u_{0}, v_{0}$ are nonnegative and suitably smooth functions with compact support.

Due to the degeneracy of the equations in (1.1), there is no classical solution to the system (1.1)-(1.3) generally and weak solutions should be considered. The local existence and the comparison principle of the weak solutions can be established; see $[6,10]$. In this paper, we investigate the large-time behaviour of solutions to the system (1.1)-(1.3), including blow-up in a finite time and global existence in time. In

Supported by the NNSF.

(C) 2012 Australian Mathematical Publishing Association Inc. 0004-9727/2012 \$16.00 
particular, we are interested in the critical global existence curve and critical Fujita curve which can be used to describe the large-time behaviour of solutions exactly.

In the wake of the pioneering work of Fujita [4], many results on critical Fujita exponents for diffusion equations with nonlinear sources can be found; see [1-5, 7-9]. It was Ferreira et al. [3] who considered the fast diffusive equation with nonlinear boundary sources:

$$
\begin{gathered}
\frac{\partial u}{\partial t}=\frac{\partial^{2} u^{m}}{\partial x^{2}}, \quad x>0, t>0, \\
-\frac{\partial u^{m}}{\partial x}(0, t)=u^{p}(0, t), \quad t>0, \\
u(x, 0)=u_{0}(x), \quad x>0,
\end{gathered}
$$

with $0<m<1$ and $p \geq 0$. They proved that the critical global existence exponent $p_{0}=(m+1) / 2$ and the critical Fujita exponent $p_{c}=m+1$. Here, $p_{0}$ and $p_{c}$ are called the critical global existence exponent and the critical Fujita exponent, respectively, if the following properties hold:

(i) if $0<p<p_{0}$, then every nontrivial nonnegative solution is global in time;

(ii) if $p_{0}<p<p_{c}$, then the nontrivial nonnegative solutions blow up in a finite time;

(iii) if $p>p_{c}$, then the solutions are global for small initial data and blow up in a finite time for large initial data.

Instead of critical exponents, there exist the critical global existence curve and the critical Fujita curve for the coupled system of diffusion equations. In [9], the authors studied the coupled system (1.1)-(1.3) in the one-dimensional case, that is,

$$
\begin{gathered}
\frac{\partial u}{\partial t}=\frac{\partial^{2} u^{m}}{\partial x^{2}}, \quad \frac{\partial v}{\partial t}=\frac{\partial^{2} v^{n}}{\partial x^{2}}, \quad x>0, t>0, \\
-\frac{\partial u^{m}}{\partial x}(0, t)=v^{p}(0, t), \quad-\frac{\partial v^{n}}{\partial x}(0, t)=u^{q}(0, t), \quad t>0, \\
u(x, 0)=u_{0}(x), \quad v(x, 0)=v_{0}(x), \quad x>0,
\end{gathered}
$$

with $0<m, n<1, p, q \geq 0$. They proved that the critical global existence curve is $p q=(m+1)(n+1) / 4$ and the critical Fujita curve is $\min \left\{\alpha_{1}+\beta_{1}, \alpha_{2}+\beta_{2}\right\}=0$, where

$$
\begin{aligned}
\alpha_{1} & =\frac{2 p+n+1}{(m+1)(n+1)-4 p q}, & \beta_{1} & =\frac{p(m-1-2 q)+(n+1) m}{(m+1)(n+1)-4 p q}, \\
\alpha_{2} & =\frac{2 q+m+1}{(m+1)(n+1)-4 p q}, & \beta_{2} & =\frac{q(n-1-2 p)+(m+1) n}{(m+1)(n+1)-4 p q} .
\end{aligned}
$$

That is to say, if $p q \leq(m+1)(n+1) / 4$, every nonnegative solution is global in time. On the other hand, if $p q>(m+1)(n+1) / 4$, then every nonnegative, nontrivial solution blows up in finite time when $\alpha_{1}+\beta_{1} \leq 0$ or $\alpha_{2}+\beta_{2} \leq 0$, while there exist both the blow-up solution with large initial data and the global solution with small initial data when $\alpha_{1}+\beta_{1}>0$ and $\alpha_{2}+\beta_{2}>0$. 
Subsequently, Pang et al. [7] dealt with the Newtonian filtration equation with nonlinear boundary source:

$$
\begin{gathered}
\frac{\partial u}{\partial t}=\Delta u^{m}, \quad(x, t) \in\left(\mathbb{R}^{N} \backslash B_{1}(0)\right) \times(0, T), \\
\nabla u^{m} \cdot \vec{v}=u^{p}(x, t), \quad(x, t) \in \partial B_{1}(0) \times(0, T), \\
u(x, 0)=u_{0}(x), \quad x \in \mathbb{R}^{N} \backslash B_{1}(0),
\end{gathered}
$$

where $0<m<1, p \geq 0$. They proved that if $N>2$, then $p_{0}=p_{c}=(m+1) / 2$.

The above works motivate us to investigate the critical curves of the system (1.1)-(1.3). In the present paper, we prove that both the critical global existence curve and the critical Fujita curve are

$$
p q=\frac{(m+1)(n+1)}{4} .
$$

It is clear that the above result is quite different from the known results obtained in [9] for the corresponding one-dimensional problem.

By virtue of the radial symmetry of the exterior domain of the unit ball as in [7], the result (1.7) can be extended to the following more general problems:

$$
\begin{gathered}
\frac{\partial}{\partial t}\left(|x|^{\lambda_{1}} u\right)=\operatorname{div}\left(|x|^{\lambda_{1}} \nabla u^{m}\right), \quad \frac{\partial}{\partial t}\left(|x|^{\lambda_{2}} v\right)=\operatorname{div}\left(|x|^{\lambda_{2}} \nabla v^{n}\right), \\
(x, t) \in\left(\mathbb{R}^{N} \backslash B_{1}(0)\right) \times(0, T), \\
\nabla u^{m} \cdot \vec{v}=v^{p}(x, t), \quad \nabla v^{n} \cdot \vec{v}=u^{q}(x, t), \quad(x, t) \in \partial B_{1}(0) \times(0, T), \\
u(x, 0)=u_{0}(x), \quad v(x, 0)=v_{0}(x), \quad x \in \mathbb{R}^{N} \backslash B_{1}(0),
\end{gathered}
$$

with $\lambda_{1}, \lambda_{2}>2-N, N \geq 1$. In other words, the phenomenon that the critical global existence curve and the critical Fujita curve coincide for the system (1.1)-(1.3) also occurs in the system (1.8)-(1.10).

Our main results are as follows.

THEOREM 1.1. If $N>2$, then the critical global existence curve and the critical Fujita curve for the system (1.1)-(1.3) are

$$
p q=\frac{(m+1)(n+1)}{4} .
$$

That is to say, if $p q \leq(m+1)(n+1) / 4$, then all nonnegative solutions to the system (1.1)-(1.3) exist globally in time; while if $p q>(m+1)(n+1) / 4$, then the nonnegative solutions to the system (1.1)-(1.3) blow up in finite time for large initial data and exist globally for small initial data.

Theorem 1.2. If $\lambda_{1}, \lambda_{2}>2-N, N \geq 1$, then the critical global existence curve and the critical Fujita curve for the system (1.8)-(1.10) are

$$
p q=\frac{(m+1)(n+1)}{4} .
$$




\section{Proofs of main results}

Since Theorem 1.1 is a special case of Theorem 1.2 (the case $\lambda_{1}=\lambda_{2}=0$ and $N>2$ ), we only prove Theorem 1.2 in this section.

Consider the problem

$$
\begin{gathered}
\frac{\partial u}{\partial t}=\frac{\partial^{2} u^{m}}{\partial r^{2}}+\frac{\tilde{\lambda}_{1}}{r} \frac{\partial u^{m}}{\partial r}, \quad \frac{\partial v}{\partial t}=\frac{\partial^{2} v^{n}}{\partial r^{2}}+\frac{\tilde{\lambda}_{2}}{r} \frac{\partial v^{n}}{\partial r}, \quad r>1, t>0, \\
-\frac{\partial u^{m}}{\partial r}(1, t)=v^{p}(1, t), \quad-\frac{\partial v^{n}}{\partial r}(1, t)=u^{q}(1, t), \quad t>0, \\
u(r, 0)=u_{0}(r), \quad v(r, 0)=v_{0}(r), \quad r>1,
\end{gathered}
$$

where $0<m, n<1, p, q \geq 0, N \geq 1$, and where $\tilde{\lambda}_{1}=\lambda_{1}+N-1>1, \tilde{\lambda}_{2}=\lambda_{2}+N-$ $1>1$, and $u_{0}, v_{0}$ are nonnegative and suitably smooth functions with compact support. We will first study the large-time behaviour of the solutions to the system (2.1)-(2.3).

Proposition 2.1. If $p q \leq(m+1)(n+1) / 4$, then all nonnegative solutions to the system (2.1)-(2.3) exist globally in time.

Proposition 2.2. If $p q>(m+1)(n+1) / 4$, then the nonnegative solutions to the problem (2.1)-(2.3) with large initial data blow up in finite time.

Remark 2.3. Propositions 2.1 and 2.2 indicate that the critical global existence curve for the system $(2.1)-(2.3)$ is $p q=(m+1)(n+1) / 4$.

PRoposition 2.4. If $p q \neq m n$, then every nonnegative nontrivial solution to the problem (2.1)-(2.3) with small initial data exists globally.

Proof of Proposition 2.1. Similarly to the proof that every nonnegative solution of the system (1.4)-(1.6) exists globally in time if $p q \leq(m+1)(n+1) / 4$ [9, Theorem 1], we define

$$
\begin{gathered}
\bar{u}(r, t)=e^{k t}\left(M+e^{-L_{1} \eta_{1}}\right)^{1 / m}, \quad \eta_{1}=(r-1) e^{k(1-m) t / 2}, \quad r>1, t>0, \\
\bar{v}(r, t)=e^{k(m+1) t /(2 p)}\left(M+e^{-L_{2} \eta_{2}}\right)^{1 / n}, \quad \eta_{2}=(r-1) e^{k(1+m)(1-n) t /(4 p)}, \quad r>1, t>0,
\end{gathered}
$$

where $L_{1}=(M+1)^{p / n}, L_{2}=(M+1)^{q / m}, M$ is large enough such that

$$
M^{1 / m}-\frac{1-m}{2 m e}(M+1)^{(1-m) / m}>0, \quad M^{1 / n}-\frac{1-n}{2 n e}(M+1)^{(1-n) / n}>0
$$

and

$$
M \geq \max \left\{\left\|u_{0}\right\|_{\infty},\left\|v_{0}\right\|_{\infty}, 1\right\},
$$

$k$ is a positive constant satisfying

$$
k\left(M^{1 / m}-\frac{1-m}{2 m e}(M+1)^{(1-m) / m}\right) \geq L_{1}^{2},
$$

and

$$
k \frac{m+1}{2 p}\left(M^{1 / n}-\frac{1-n}{2 n e}(M+1)^{(1-n) / n}\right) \geq L_{2}^{2} .
$$


Noticing the global existence in time of $(\bar{u}, \bar{v})$, we get that the solution of the problem (2.1)-(2.3) exists globally by the comparison principle. The proof is complete.

Proof of Proposition 2.2. The proposition is proved by constructing a kind of lower blow-up solution. Set

$$
\begin{array}{lll}
\underline{u}(r, t)=(T-t)^{-\alpha_{1}} f_{1}\left(\eta_{1}\right), & \eta_{1}=(r-1)(T-t)^{-\beta_{1}}, & r>1,0<t<T, \\
\underline{v}(r, t)=(T-t)^{-\alpha_{2}} f_{2}\left(\eta_{2}\right), & \eta_{2}=(r-1)(T-t)^{-\beta_{2}}, & r>1,0<t<T,
\end{array}
$$

where $T>0$,

$$
\begin{array}{ll}
\alpha_{1}=\frac{1+2 p+n}{4 p q-(m+1)(n+1)}, & \beta_{1}=\frac{1+\alpha_{1}(1-m)}{2}, \\
\alpha_{2} & =\frac{1+2 q+m}{4 p q-(m+1)(n+1)},
\end{array}
$$

Assume that $f_{1}, f_{2}$ satisfy

$$
f_{1}\left(\eta_{1}\right), f_{2}\left(\eta_{2}\right) \geq 0, \quad f_{1}\left(\eta_{1}\right)^{\prime}, f_{2}\left(\eta_{2}\right)^{\prime} \leq 0, \quad f_{1}\left(\eta_{1}\right)^{\prime \prime}, f_{2}\left(\eta_{2}\right)^{\prime \prime} \geq 0, \quad \eta_{1}, \eta_{2} \geq 0 .
$$

After some computations, we can see that $(\underline{u}, \underline{v})$, with $\underline{u}(r, 0) \leq u_{0}(r), \underline{v}(r, 0) \leq v_{0}(r)$, is a lower solution to the problem (2.1)-(2.3), if the following inequalities hold:

$$
\begin{gathered}
\left(f_{1}^{m}\left(\eta_{1}\right)\right)^{\prime \prime}+\tilde{\lambda}_{1} T^{\beta_{1}}\left(f_{1}^{m}\left(\eta_{1}\right)\right)^{\prime}-\alpha_{1} f_{1}\left(\eta_{1}\right) \geq 0, \quad \eta_{1} \geq 0 \\
\left(f_{2}^{n}\left(\eta_{2}\right)\right)^{\prime \prime}+\tilde{\lambda}_{2} T^{\beta_{2}}\left(f_{2}^{n}\left(\eta_{2}\right)\right)^{\prime}-\alpha_{2} f_{2}\left(\eta_{2}\right) \geq 0, \quad \eta_{2} \geq 0 \\
-\left(f_{1}^{m}\right)^{\prime}(0) \leq f_{2}^{p}(0) \\
-\left(f_{2}^{n}\right)^{\prime}(0) \leq f_{1}^{q}(0) .
\end{gathered}
$$

Take

$$
\begin{array}{cc}
f_{1}\left(\eta_{1}\right)=\left(\left(A_{1}+B_{1} \eta_{1}\right)^{2 m /(m-1)}-\left(2 A_{1}\right)^{2 m /(m-1)}\right)_{+}^{1 / m}, & \eta_{1} \geq 0, \\
f_{2}\left(\eta_{2}\right)=\left(\left(A_{2}+B_{2} \eta_{2}\right)^{2 n /(n-1)}-\left(2 A_{2}\right)^{2 n /(n-1)}\right)_{+}^{1 / n}, & \eta_{2} \geq 0
\end{array}
$$

where $0<A_{1}, A_{2}<1, B_{1}, B_{2}>0$ are the constants to be determined. It is clear that the above $f_{1}, f_{2}$ satisfy (2.4). We now verify that $f_{1}, f_{2}$ satisfy (2.5)-(2.8). We claim that (2.5) is valid for $0<\eta_{1}<A_{1} / B_{1}$. In fact, we only need to verify that

$$
\frac{1}{2}\left(f_{1}^{m}\left(\eta_{1}\right)\right)^{\prime \prime} \geq-\tilde{\lambda}_{1} T_{1}^{\beta}\left(f_{1}^{m}\left(\eta_{1}\right)\right)^{\prime}, \quad \frac{1}{2}\left(f_{1}^{m}\left(\eta_{1}\right)\right)^{\prime \prime} \geq \alpha_{1} f_{1}\left(\eta_{1}\right)
$$

that is,

$$
\frac{1+m}{1-m} B_{1} \geq 2 \tilde{\lambda}_{1} T^{\beta_{1}}\left(A_{1}+B_{1} \eta_{1}\right), \quad B_{1}^{2} \frac{m(m+1)}{(1-m)^{2}} \geq \alpha_{1} .
$$

Let $B_{1}=\sqrt{\alpha_{1}(1-m)^{2} / m(m+1)}+1$. Thus, (2.11) is valid if $A_{1}$ is small enough that

$$
\frac{1+m}{1-m} B_{1} \geq 4 \tilde{\lambda}_{1} T^{\beta_{1}} A_{1}
$$


Similarly, (2.6) is valid with $B_{2}=\sqrt{\alpha_{2}(1-n)^{2} / n(n+1)}+1$ and $A_{2}$ small enough that $(1+n) B_{2} /(1-n) \geq 4 \tilde{\lambda}_{2} T^{\beta_{2}} A_{2}$.

Substituting (2.9) and (2.10) into (2.7) and (2.8) yields

$$
\begin{aligned}
& \frac{2 m}{1-m} A_{1}^{(m+1) /(m-1)} B_{1} \leq\left(1-2^{2 n /(n-1)}\right)^{p / n} A_{2}^{2 p /(n-1)}, \\
& \frac{2 n}{1-n} A_{2}^{(n+1) /(n-1)} B_{2} \leq\left(1-2^{2 m /(m-1)}\right)^{q / m} A_{1}^{2 q /(m-1)} .
\end{aligned}
$$

Since $p q>(m+1)(n+1) / 4$, there exists a constant $\sigma$, such that

$$
\frac{(m+1)(n-1)}{2 p(m-1)}<\sigma<\frac{2 q(n-1)}{(n+1)(m-1)} .
$$

Choosing $A_{2}=A_{1}^{\sigma}$ and taking $0<A_{1}<1$ sufficiently small,

$$
\begin{aligned}
& \frac{2 m}{1-m} A_{1}^{(m+1) /(m-1)} B_{1} \leq\left(1-2^{2 n /(n-1)}\right)^{p / n} A_{1}^{2 \sigma p /(n-1)}, \\
& \frac{2 n}{1-n} A_{1}^{\sigma(n+1) /(n-1)} B_{2} \leq\left(1-2^{2 m /(m-1)}\right)^{q / m} A_{1}^{2 q /(m-1)} .
\end{aligned}
$$

This proves inequalities (2.12) and (2.13).

Therefore, the solution $(u, v)$ of the problem (2.1)-(2.3) blows up in a finite time if $\left(u_{0}(r), v_{0}(r)\right)$ is large enough such that

$$
u_{0}(r) \geq \underline{u}(r, 0), \quad v_{0}(r) \geq \underline{v}(r, 0), \quad r>1 .
$$

The proof is complete.

Proof of Proposition 2.4. The proof is completed by constructing the global upper solution

$$
\bar{u}(r, t)=\left(B_{1} r^{1-\tilde{\lambda}_{1}}\right)^{1 / m}, \quad \bar{v}(r, t)=\left(B_{2} r^{1-\tilde{\lambda}_{2}}\right)^{1 / n}, \quad r>1, t>0,
$$

where

$$
\begin{gathered}
B_{1}=\left(\tilde{\lambda}_{1}-1\right)^{-m n /(m n-p q)}\left(\tilde{\lambda}_{2}-1\right)^{-m p /(m n-p q)}, \\
B_{2}=\left(\tilde{\lambda}_{1}-1\right)^{-n q /(m n-p q)}\left(\tilde{\lambda}_{2}-1\right)^{-m n /(m n-p q)} .
\end{gathered}
$$

A simple calculation shows that

$$
\begin{aligned}
& \frac{\partial \bar{u}}{\partial t}-\frac{\partial^{2} \bar{u}^{m}}{\partial r^{2}}-\frac{\tilde{\lambda}_{1}}{r} \frac{\partial \bar{u}^{m}}{\partial r}=0, \quad r>1, t>0, \\
& \frac{\partial \bar{v}}{\partial t}-\frac{\partial^{2} \bar{v}^{n}}{\partial r^{2}}-\frac{\tilde{\lambda}_{2}}{r} \frac{\partial \bar{v}^{n}}{\partial r}=0, \quad r>1, t>0, \\
& -\frac{\partial \bar{u}^{m}}{\partial r}(1, t)=\bar{v}^{p}(1, t), \quad t>0, \\
& -\frac{\partial \bar{v}^{n}}{\partial r}(1, t)=\bar{u}^{q}(1, t), \quad t>0 .
\end{aligned}
$$


By the comparison principle, for any initial data $\left(u_{0}, v_{0}\right)$ which is small enough to satisfy

$$
u_{0}(r) \leq \bar{u}(r, 0), \quad v_{0}(r) \leq \bar{v}(r, 0), \quad r>1,
$$

the solution of the problem (2.1)-(2.3) exists globally in time.

Proof of Theorem 1.2. Since the functions $u_{0}(x), v_{0}(x)$ have compact supports, we can choose two radially symmetric and bounded functions, denoted by $u_{1}(x)=u_{1}(|x|) \geq$ $u_{0}(x), v_{1}(x)=v_{1}(|x|) \geq v_{0}(x)$. By Proposition 2.1 and the comparison principle, we can establish global existence of solutions for the problem (1.8)-(1.10) with $p q<$ $(m+1)(n+1) / 4$. For $p q>(m+1)(n+1) / 4$, if the large and radially symmetric initial data satisfy $u_{0}(x) \geq \underline{u}(|x|, 0), v_{0}(x) \geq \underline{v}(|x|, 0)$, then the solutions to the system (1.8)-(1.10) blow up by the comparison principle and Proposition 2.2. This shows that the critical global existence curve is $p q=(m+1)(n+1) / 4$ for the system $(1.8)-(1.10)$.

On the other hand, using the comparison principle again, we see that the solution $(u, v)$ of (1.8)-(1.10) with

$$
u_{0}(x) \leq\left(B_{1}|x|^{2-\lambda_{1}-N}\right)^{1 / m}, \quad v_{0}(x) \leq\left(B_{2}|x|^{2-\lambda_{2}-N}\right)^{1 / m},
$$

where

$$
\begin{gathered}
B_{1}=\left(\lambda_{1}+N-2\right)^{-m n /(m n-p q)}\left(\lambda_{2}+N-2\right)^{-m p /(m n-p q)}, \\
B_{2}=\left(\lambda_{1}+N-2\right)^{-n q /(m n-p q)}\left(\lambda_{2}+N-2\right)^{-m n /(m n-p q)},
\end{gathered}
$$

exists globally for $p q \neq m n$ from Proposition 2.4. This combined with Proposition 2.2 establishes the critical Fujita curve $p q=(m+1)(n+1) / 4$ for the system (1.8)-(1.10).

\section{Acknowledgements}

We would like to express our many thanks to the editor and reviewers for their constructive suggestions to improve the previous version of this paper.

\section{References}

[1] K. Deng, M. Fila and H. A. Levine, 'On critical exponent for a system of heat equations coupled in the boundary conditions', Acta Math. Univ. Comenian. 63(2) (1994), 169-192.

[2] K. Deng and H. A. Levine, 'The role of critical exponents in blow-up theorems: the sequel', J. Math. Anal. Appl. 243(1) (2000), 85-126.

[3] R. Ferreira, A. Pablo, F. de Quirós and J. D. Rossi, 'The blow-up profile for a fast diffusion equation with a nonlinear boundary condition', Rocky Mountain J. Math. 33 (2003), 123-146.

[4] H. Fujita, 'On the blowing up of solutions of the Cauchy problem for $u_{t}=\Delta u+u^{1+\alpha}, J$. Fac. Sci. Univ. Tokyo Sect. I 13 (1966), 109-124.

[5] V. A. Galaktionov and H. A. Levine, 'A general approach to critical Fujita exponents in nonlinear parabolic problems', Nonlinear Anal. 34(7) (1998), 1005-1027.

[6] A. S. Kalashnikov, 'Some problems of the qualitative theory of nonlinear degenerate second-order parabolic equations', Russian Math. Surveys 42(2) (1987), 169-222. 
[7] P. Y. H. Pang, Z. J. Wang and J. X. Yin, 'Critical exponents for nonlinear diffusion equations with nonlinear boundary sources', J. Math. Anal. Appl. 343(2) (2008), 654-662.

[8] F. de Quirós and J. D. Rossi, 'Blow-up sets and Fujita type curves for a degenerate parabolic system with nonlinear boundary conditions', Indiana Univ. Math. J. 50 (2001), 629-654.

[9] Z. J. Wang, Q. Zhou and W. Q. Lou, 'Critical exponents for porous medium systems coupled via nonlinear boundary flux', Nonlinear Anal. 7(1) (2009), 2134-2140.

[10] Z. Q. Wu, J. N. Zhao, J. X. Yin and H. L. Li, Nonlinear Diffusion Equations (World Scientific, River Edge, NJ, 2001).

RUNMEI DU, Institute of Mathematics, Jilin University, Changchun 130012, PR China

ZEJIA WANG, College of Mathematics, Jilin University, Changchun 130012, PR China

e-mail: matwzj@jlu.edu.cn 